\title{
On (1,2)- Blast Domination Number for Some Total Graphs
}

\author{
P.Rajakumari, K. Ameenal Bibi
}

\begin{abstract}
The hub of this article is a search on the behavior of the $(1,2)$ Blast domination number for total graphs of some particular graphs.
\end{abstract}

Key Words - Connected domination number, Comb graph, $(1,2)$ domination number, Helm graph, Total graph of a graph and, Wheel graph.

\section{INTRODUCTION [5]}

Let $G=(V, E)$ be a simple finite, undirected and connected graph. Generally, for a graph $G$ we denote $V(G), E(G), \Delta(G)$ and $\delta(G)$ for its vertex set, edge set, maximum and minimum degree respectively. Degree of a vertex $v$ is denoted by $d(v)$. For any graph theoretic terminology, we refer Chartrand and Lesniak [2].

Domination in graphs has become an important area of research in graphs as evidenced by the many results contained in two books by Haynes, Hedetniemi and Slater (1998) [4]. A variety of connectedness has been studied in the literature by considering the existence of a path between any two vertices. A communication network in which a communicating node can send a message to two stations at one stretch will be more effective and economic. Such an optimization leads to the concept of triple connected graphs. The concept of triple connected graphs was introduced by Paulraj Joseph et.al [8].

Murugesan et.al introduced the $(1,2)$ domination in graphs. They also introduced the line, middle and central graphs of path $P_{n}$, cycle $C_{n}$ and star $K_{1, n}$ graphs. The related ideas regarding these graphs can be seen in [10] [11]. Vernold Vivin and Venkatachalam introduced some wheel graph families of Total graphs.

G. Mahadevan et.al introduced the concept of Blast domination number of a graph with real life application and also introduced the Blast domination number for central and total graph of star (2017) and $\vartheta$ - obrazom (2018) graphs. Thus we have done a search on the behavior of our new parameter over the total graph of a variety of the graphs.

\section{PRELIMINARIES}

Definition 2.1 [12]

The $n$-Sunlet graph of $2 n$ vertices is obtained by attaching $n$ pendant edges to the cycle $C_{n}$ and is denoted by $S_{n}$.

Definition 2.2 [6]

Revised Manuscript Received on July 18, 2019.

P.Rajakumari, Department of Mathematics, D.K.M.College for Women (Autonomous), Vellore-632001, Tamilnadu, India (rajakumari0990@gmail.com)

K. Ameenal Bibi, Department of Mathematics, D.K.M.College for Women (Autonomous), Vellore-632001, Tamilnadu, India.
For any integer $n \geq 4$, a Wheel graph $W_{n}$ (i.e, cone of a cycle) of order $n+1, n$-wheel is a graph that contains a cycle of order $n-1$, for which every vertex in the cycle is connected to one new vertex.

\section{Definition 2.3 [1]}

The helm graph $H_{n}$ is the graph obtained from an $n$-wheel graph by joining a pendant edge at each vertices of the cycle. Let $V\left(H_{n}\right)=\{v\} \cup\left\{v_{i}: 0 \leq i \leq n-1\right\} \cup\left\{u_{i}\right.$ : $0 \leq i \leq n-1\}, E\left(H_{n}\right)=\left\{e_{i}=v_{i} v_{i+1}: 0 \leq i \leq n-1\right.$, subscript modulo $n\} \cup\left\{e^{\prime}{ }_{i}=v v_{i}: 0 \leq i \leq n-1\right\} \cup$ $\left\{e_{i} "=v_{i} u_{i}: 0 \leq i \leq n-1\right\}$.

\section{Definition 2.4}

A Comb graph is a Caterpillar in which each vertex in the path is joined to exactly one pendent vertex.

\section{Definition 2.5 [6]}

A graph $G$ is said to be triple connected, if any three vertices of $G$ lie on a path.

\section{Definition 2.6 [6]}

A non-empty subset $D$ of $V$ of vertices in a graph $G=$ $(V, E)$ is said to be a dominating set, if each vertices, $v$ in $V$ is either an element of $D$ or it is adjacent to an element of $D$. The domination number is the minimum cardinality taken over all such connected dominating sets of $G$.

\section{Definition 2.7 [6]}

A non-empty subset $D$ of $V$ of vertices in a graph, $G=(V, E)$ is called a connected dominating set, if every vertex of $\langle V-D>$ is adjacent to at least one vertex in $D$ and the induced sub graph $\langle D\rangle$ is connected. The connected domination number is the minimum cardinality taken over all such connected dominating sets of $G$.

\section{Definition 2.8 [6]}

A non-empty subset $D$ of $V$ of a connected graph $G$ is called a Blast dominating set (or) BD-set, if $D$ is a connected dominating set and the induced sub graph $\langle V-D\rangle$ is triple connected. The minimum cardinality taken over all such Blast dominating sets is called the Blast domination number of $G$ and is denoted by $\gamma_{c}^{t c}(G)$.

Definition 2.9 [7]

A non-empty subset $D \subseteq V$ is called a $(1,2)$ dominating set in a graph $G=(V, E)$ having the property that for every vertex $v$ in $V-D$ there is atleast one vertex in $D$ at distance 1 from $v$ and a second vertex in $D$ at distance 
atmost 2 from $v$.The order of the smallest $(1,2)$ - dominating set of $G$ is called the $(1,2)$ - domination number of $G$ denoted by $\gamma_{(1,2)}(G)$.

\section{Definition 2.10[12]}

Let $G$ be a graph with vertex set $V(G)$ and edge set $E(G)$. The total graph [3] of $G$, denoted by $T(G)$ is defined in the following way. The vertex set of $T(G)$ is $V(G) \cup E(G)$ and Two vertices $x, y$ of $T(G)$ are adjacent in $T(G)$, if either

(i) $x, y$ in $V(G)$ and $x$ is adjacent to $y$ in $G$ (or)

(ii) $x, y$ in $E(G)$ and $x, y$ are adjacent in $G$ (or)

(iii) $x$ is in $V(G), y$ is in $E(G)$, and $x, y$ are incident in $G$.

\section{MAIN RESULT}

\section{(1,2)- Blast Domination Number for Some Total Graphs}

In this section $(1,2)$ - Blast domination number of total graphs of some special classes of graphs are obtained also exact values for some standard graphs discussed.

\section{Definition 3.1}

A non-empty subset $D$ of $V$ of a graph $G=(V, E)$ is said to be $(1,2)$ - Blast dominating set having property that, for every vertex $v$ in $V-D$, there is at least one vertex in $D$ at distance 1 from $v$ and a second vertex in $D$ at distance almost 2 from $v$ and also $\langle D\rangle$ is a connected dominating set, the induced sub graph $\langle V-D\rangle$ is triple connected. The minimum cardinality taken over all minimal $(1,2)$ Blast dominating set is called the $(1,2)$ - Blast domination number and is denoted by $\gamma_{(1,2) c}^{t c}(G)$.

\section{Theorem 3.2}

$$
\text { For any Path } P_{n}(n \geq 3), \gamma_{(1,2) c}^{t c}\left[T\left(P_{n}\right)\right]=n-1
$$

\section{Proof}

Let $P_{n}=\left\{v_{1}, v_{2}, \ldots v_{n}\right\}$ and $e_{i}=\left\{v_{i} v_{i+1}: 1 \leq i \leq n-\right.$ $1\}$. By the definition of total graph, $T\left(P_{n}\right)$ can be obtained by subdividing each edge of $P_{n}$ exactly once in $T\left(P_{n}\right)$ and joining all these middle vertices of adjacent edges of $P_{n}$ and also joining the adjacent vertices of $P_{n}$. Let $u_{i} \in V\left(T\left(P_{n}\right)\right)$ be the vertex corresponding to $e_{i}$ and

$$
=\left\{v_{i} \cup e_{i}: 1 \leq i \leq n\right\}
$$

$$
V\left[T\left(P_{n}\right)\right]=V\left(P_{n}\right) \cup E\left(P_{n}\right)
$$

In which the vertices $\left\{e_{i}: 1 \leq i \leq n\right\}$ induces a path of order $n-1$ and the vertices adjacent to $\left\{v_{i}: 1 \leq i \leq n\right\}$. Since we need a connected dominating set, so, let us presume $D=\left\{e_{i}: 1 \leq i \leq n\right\}$ dominates the vertices $v_{i}$ of $T\left(P_{n}\right)$ and simultaneously its complement $\langle V-D\rangle$ is triple connected in $T\left(P_{n}\right)$.Thus we get our blast dominating set. Hence $\gamma_{c}^{t c}\left(T\left(P_{n}\right)\right)=n-1$

In $T\left(P_{n}\right)$ we can see that every vertex in $V-D$ there is a vertex $v_{i}$ which is the first vertex of distance 1 and a second vertex $e_{i}$ which is of at distance 2. Clearly, it forms a $(1,2)$ dominating set.

Now the presume result $D=\left\{e_{i}: 1 \leq i \leq n\right\}$ holds for $T\left(P_{n}\right)$. Therefore, the minimum $(1,2)$ - Blast dominating set of the total graph of Path $P_{n}$ is $n-1$.

\section{Result 3.3}

Let $G$ be the total graph of the path graph of length $n,(n \geq 3)$. Then,
(i) $\quad \mathrm{i}\left[\mathrm{T}\left(\mathrm{P}_{\mathrm{n}}\right)\right]=\frac{\mathrm{n}+1}{2}$
(ii) $\quad$ (ii) $\Delta\left[\mathrm{T}\left(\mathrm{P}_{\mathrm{n}}\right)\right]=4$
(iii) (iii) $\delta\left[\mathrm{T}\left(\mathrm{P}_{\mathrm{n}}\right)\right]=2$

Observation 3.4

(i) Every $\gamma_{c}^{t c}\left[T\left(P_{n}\right)\right]$ is an $(1,2)$ dominating set but converse is not true.

(ii) Every $\gamma_{c}^{t c}\left[T\left(P_{n}\right)\right]$ is not an equitable dominating set but $T\left(P_{n}(V-D)\right)$ is an equitable dominating set.

Theorem 3.5

For any Cycle $C_{n}(n \geq 4), \gamma_{(1,2) c}^{t c}\left[T\left(C_{n}\right)\right]=n$

Proof

Let $C_{n}$ be the cycle graph of order $n-3$ with vertices $u_{1}, u_{2}, \ldots, u_{n}$. Let $v_{i}=u_{i} u_{i+1}(1 \leq i \leq n-1)$ be the edges of cycle $C_{n}$. By the definition of total graph, $T\left(C_{n}\right)$ can be obtained by subdividing each edge $v_{i}=u_{i} u_{i+1}(1 \leq i \leq$ $n-1)$ of $C_{n}$ exactly once in $T\left(C_{n}\right)$ and joining all these middle vertices of adjacent edges of $C_{n}$ and also joining the adjacent vertices of $C_{n}$. Let the vertices of $T\left(C_{n}\right)$ be labeled by

$$
\begin{gathered}
{\left[T\left(C_{n}\right)\right]=V\left(C_{n}\right) \cup E\left(C_{n}\right)} \\
=\left\{u_{i} v_{i}: 1 \leq i \leq n\right\} \cup\left\{u_{i} u_{i+1}, v_{j} v_{j+1}, u_{i} v_{i}, v_{j} u_{i+1}: 1 \leq\right.
\end{gathered}
$$
$i \leq n-1,1 \leq j \leq n-2\}$ and since $|V(G)|=2 n$ and $|E(G)|=4 n$,in which the set of vertices $\left\{u_{i}: 1 \leq i \leq n\right\}$ induces a cycle graph of order $\mathrm{n}$ also $v_{i}$ induces a cycle of order $n$.

Consider $D=\left\{v_{i}: 1 \leq i \leq n\right\}$. We get the blast dominating set whose induced sub graph $\langle D\rangle$ is connected and simultaneously its complement $\langle V-D\rangle$ is triple connected in $T\left(C_{n}\right)$. Thus, $\gamma_{c}^{t c}\left(T\left(C_{n}\right)\right)=n$.

In $T\left(C_{n}\right)$ we note that every vertex in $\langle V-D\rangle$ there exists a first vertex $v_{i}$ which is of distance 1 and a second vertex $v_{i+1}$ is of at distance 2 . Clearly, it forms a $(1,2)$ dominating set. Now the presume result $D=\left\{v_{i}: 1 \leq i \leq\right.$ $n$ \} holds for $T\left(C_{n}\right)$. Therefore the minimum $(1,2)$ - Blast dominating set of total graph of cycle $C_{n}$ is $n$.

\section{Result 3.6}

Let $G$ be the total graph of the Cycle graph of order $n, n \geq$ 4. Then,

(ii) $\Delta\left[\mathrm{T}\left(\mathrm{C}_{\mathrm{n}}\right)\right]=4=\delta\left[\mathrm{T}\left(\mathrm{C}_{\mathrm{n}}\right)\right]$

$$
\text { (i) } \mathrm{i}\left[\mathrm{T}\left(\mathrm{C}_{\mathrm{n}}\right)\right]=\frac{\mathrm{n}}{2}
$$

\section{Observation 3.7}

Every total graph of cycle graph is an equitable dominating set if and only if $\langle V-D\rangle$ is also an equitable dominating set.

\section{Theorem 3.8}

For any Wheel $\mathrm{W}_{\mathrm{n}}(n \geq 3), \gamma_{(1,2) c}^{t c}\left[T\left(W_{n}\right)\right]=n$.

\section{Proof}

Let $V\left(W_{n}\right)=\left\{v, v_{1}, v_{2}, \ldots, v_{n-1}\right\}$ and let $V\left(T\left(W_{n}\right)\right)=$ $\left\{v \cup\left\{v_{i}: 1 \leq i \leq n\right\} \cup\left\{u_{i}: 1 \leq i \leq n\right\} \cup\left\{e_{i}: 1 \leq i \leq n\right\}\right.$ where $u_{i}$ is the vertex of $T\left(W_{n}\right)$ corresponding to the edge 
$v_{i} v_{i+1}$ of $W_{n}(1 \leq i \leq n-1)$. The apex $v$ adjacent to all ther spokes $e_{i}(1 \leq i \leq n)$ and the vertices $v_{i}$ of $T\left(W_{n}\right)$ except $v_{i}$. Let us choose and start with any one of the vertices corresponding to the spoke edges in $W_{n}$. Now fix $v_{i}$, clearly $v_{i}$ is adjacent to all the vertices of $T\left(W_{n}\right)$. Since $D=\left\{v_{i}\right\}$, dominates all the vertices of $T\left(W_{n}\right)$ whose $\langle D\rangle$ is connected and simultaneously, its complement $<V-$ $D>$ is triple connected in $T\left(W_{n}\right)$. Hence $\gamma_{c}^{t c}\left[T\left(W_{n}\right)\right]=n$.

From $T\left(W_{n}\right)$, let us presume the apex $v \cup\left\{u_{i}\right\}$ adjacent to the spoke $e_{i}: 1 \leq i \leq n$ and other vertices of $\left\{e_{i} \cup v_{i}\right\}$. Here the spoke $e_{i}$ there is a first vertex $v$ is of distance 1 and second vertex $u_{i}$ is of distance 2 . Also each vertex $v_{i}$ the there is a first vertex $u_{i}$ in $D$ is of distance 1 and second vertex $v$ in $D$ is of distance 2. Clearly, the set $\left\{e_{i} \cup v_{i}\right\}$ it forms a $(1,2)$ dominating set but the induced graph $<v \cup$ $\left\{u_{i}\right\}>$ is disconnected. Therefore, $\left\langle v \cup\left\{u_{i}\right\}>\right.$ is not a blast dominating set. Now, again choose and start with the vertex $v_{i}$. From the apex $v$ the first vertex $v_{i}$ is of distance 1 and second vertex $v_{i+1}$ is of distance atmost 2 . It forms a $(1,2)$ - dominating set which is triple connected. By the presumed condition, clearly it will form a $(1,2)$-blast dominating set. Therefore, $\gamma_{(1,2) c}^{t c}\left[T\left(W_{n}\right)\right]=n$.

\section{Result 3.9}

For the total graph of Wheel graph of order $n,(n \geq 3)$. Then

$$
\text { (i) } \begin{gathered}
i\left[T\left(W_{n}\right)\right]=2(i i) \Delta\left[T\left(W_{n}\right)\right]=2 n \\
(i i i) \delta\left[T\left(W_{n}\right)\right]=n+1 .
\end{gathered}
$$

Observation 3.10

If $\mathrm{D}$ is $(1,2)$ dominating set in $\mathrm{T}\left(\mathrm{W}_{\mathrm{n}}\right)$ if and only if $<V-D>$ is also $(1,2)$ dominating set in $\mathrm{T}\left(\mathrm{W}_{\mathrm{n}}\right)$.

Theorem 3.11

For any Sunlet graph $\mathrm{S}_{\mathrm{n}}(\mathrm{n} \geq 3), \gamma_{(1,2) c}^{t c}\left[T\left(S_{\mathrm{n}}\right)\right]=n$ Proof

Let $\mathrm{V}\left(\mathrm{S}_{\mathrm{n}}\right)=\mathrm{v}_{\mathrm{i}} \cup \mathrm{u}_{\mathrm{i}}: 1 \leq \mathrm{i} \leq \mathrm{n}$, and $\mathrm{E}\left(\mathrm{S}_{\mathrm{n}}\right)=\left\{\mathrm{e}_{\mathrm{i}}^{\prime}: 1 \leq \mathrm{i} \leq\right.$ $\mathrm{n}\} \cup\left\{\mathrm{e}_{\mathrm{i}}: 1 \leq \mathrm{i} \leq \mathrm{n}\right\} \cup\left\{\mathrm{e}_{\mathrm{n}}\right\}$. By the definition of total graph, $\mathrm{V}\left(\mathrm{T}\left(\mathrm{S}_{\mathrm{n}}\right)\right)=\left\{\mathrm{v}_{\mathrm{i}} \cup \mathrm{v}_{\mathrm{i}}{ }^{\prime} \cup \mathrm{u}_{\mathrm{i}}^{\prime} \cup \mathrm{u}_{\mathrm{i}}: 1 \leq \mathrm{i} \leq \mathrm{n}\right\}$ be the vertices of $\mathrm{T}\left(\mathrm{S}_{\mathrm{n}}\right)$. The vertices $\mathrm{v}_{\mathrm{i}}$ induces a cycle of order. The set of vertices $\left\{\mathrm{v}_{\mathrm{i}}^{\prime}: 1 \leq \mathrm{i} \leq \mathrm{n}\right\}$ forms a cycle with each vertex joining any two edges $\left\{\mathrm{u}_{\mathrm{i}}^{\prime}, \mathrm{u}_{\mathrm{i}+1}^{\prime}\right\}$ to the apex $\mathrm{u}_{\mathrm{i}}$ which are independent. Since the set of vertices $\left\{\mathrm{u}_{\mathrm{i}}, \mathrm{u}_{\mathrm{i}}^{\prime}, \mathrm{v}_{\mathrm{i}}^{\prime}: 1 \leq \mathrm{i} \leq\right.$ $\mathrm{n}\}$ is adjacent to $\mathrm{v}_{\mathrm{i}}$ in $\mathrm{T}\left(S_{n}\right)$.

Since we need a connected dominating set so, let us presume $\mathrm{D}=\mathrm{v}_{\mathrm{i}},(1 \leq \mathrm{i} \leq \mathrm{n}),\langle D\rangle \quad$ is connected dominating set in and simultaneously, whose $\langle V-D\rangle$ is triple connected in $T\left(S_{n}\right)$. In $\mathrm{T}\left(\mathrm{S}_{\mathrm{n}}\right)$, fix $\mathrm{v}_{\mathrm{i}}^{\prime}$ then there is a vertex $\mathrm{v}_{\mathrm{i}}$ which is of distance 1 and second vertex $\mathrm{v}_{\mathrm{i}+1}$ is at distance 2. Successively, fix the apex $\mathrm{u}_{\mathrm{i}}$ and $\mathrm{u}_{\mathrm{i}}^{\prime}$ then there is a vertex $\mathrm{v}_{\mathrm{i}}$ at distance 1 and second vertex of distance atmost 2. Clearly $\mathrm{v}_{\mathrm{i}}$ will be a $(1,2)$ - dominating set in $\mathrm{T}\left(\mathrm{S}_{\mathrm{n}}\right)$. Now the presume result $\mathrm{D}=\mathrm{v}_{\mathrm{i}},(1 \leq \mathrm{i} \leq \mathrm{n})$ holds. Therefore, minimum $(1,2)$ - Blast dominating set of total graph of Sunlet graph is $n$.

Result 3.12

For the total graph of Sunlet graph of order $(\mathrm{n} \geq 3)$. Then

(iii) $\delta\left(\mathrm{T}\left(\mathrm{S}_{\mathrm{n}}\right)\right)=2$

\section{Observation 3.13}

(i) Every $\gamma_{(1,2) c}^{t c}\left[T\left(S_{n}\right)\right]$ is a blast dominating set but $<V-D>$ can't form a blast dominating set.

(ii)Also $\mathrm{T}\left(\mathrm{S}_{\mathrm{n}}\right)$, it forms a $(1,2)$ dominating set which is not always blast dominating set in $\mathrm{T}\left(\mathrm{S}_{\mathrm{n}}\right)$.

Theorem 3.14

For any Helm graph $\mathrm{H}_{\mathrm{n}}(n \geq 3), \gamma_{(1,2) c}^{t c}\left[T\left(H_{n}\right)\right]=n$

Proof

Let $\mathrm{E}\left(\mathrm{T}\left(\mathrm{H}_{\mathrm{n}}\right)\right)=\left\{\mathrm{ve}_{\mathrm{i}}: 1 \leq \mathrm{i} \leq \mathrm{n}\right\} \cup\left\{\mathrm{e}_{\mathrm{i}}^{\prime}, \mathrm{e}_{\mathrm{i}}^{\prime \prime}: 1 \leq \mathrm{i} \leq \mathrm{n}\right\} \cup$ $\left\{\mathrm{u}_{\mathrm{i}} \mathrm{v}_{\mathrm{i}}: 1 \leq \mathrm{i} \leq \mathrm{n}\right\} \cup\left\{\mathrm{vv}_{\mathrm{i}}: 1 \leq \mathrm{i} \leq \mathrm{n}\right\} \cup\left\{\mathrm{v}_{\mathrm{i}} \mathrm{v}_{\mathrm{i}+1}: 1 \leq \mathrm{i} \leq\right.$ $\mathrm{n}$ subscript modulo $\mathrm{n}\}$. For each $\mathrm{i}, 1 \leq \mathrm{i} \leq \mathrm{n}\}$ the set of vertices $\left\{\mathrm{e}_{\mathrm{i}}^{\prime}, \mathrm{v}_{\mathrm{i}+1}, \mathrm{e}_{\mathrm{i}+1}, \mathrm{e}_{\mathrm{i}+1}^{\prime}, \mathrm{e}_{\mathrm{i}+1}^{\prime \prime}\right\}$ of $T\left(H_{n}\right)$ induces a clique of order 5. Also the set of vertices $\left\{\left\{e_{i}^{\prime}: 1 \leq i \leq n\right\} \cup v\right\}$ induces a clique $K_{n+1}$ of order $n+1$. Since the apex $v$ is adjacent to all the spokes $e_{i}^{\prime}$ and the vertex $v_{i}$. Let us choose $v_{i}$ which dominates all the vertices $\left\{v_{i+1}, e_{i}^{\prime \prime}, u_{i}, e_{i}^{\prime}, v, e_{i}\right\}$ of $T\left(H_{n}\right)$ which is connected. Let us consider $=v_{i}$, whose $<$ $D>$ is connected dominating set and simultaneously its complement $\langle V-D\rangle$ is triple connected in $T\left(H_{n}\right)$. Thus $\gamma_{c}^{t c}\left(T\left(H_{n}\right)\right)=n$.

In $T\left(H_{n}\right)$ we see that, let us fix apex $v$ there is the first vertex $\mathrm{v}_{\mathrm{i}}$ is that of distance one and second vertex $\mathrm{v}_{\mathrm{i}+1}$ is at distance atmost 2. Consequently, let us fix $\left\{\mathrm{e}_{\mathrm{i}}^{\prime}, \mathrm{e}_{\mathrm{i}}, \mathrm{e}_{\mathrm{i}}^{\prime \prime}, \mathrm{u}_{\mathrm{i}}\right\}$ in $<V-D>$ there is atleast one vertex is of distance atmost 2 in D. It form a $(1,2)$ blast dominating set. Now the presume result $\mathrm{D}=\mathrm{v}_{\mathrm{i}},(1 \leq \mathrm{i} \leq \mathrm{n})$ holds. Therefore, the minimum $(1,2)$ - Blast dominating set of total graph of Helm graph is $n$.

\section{Result 3.15}

For any total graph of Helm graph of order $(n \geq 3)$. Then

(iv) $\beta\left[\mathrm{T}\left(\mathrm{H}_{\mathrm{n}}\right)\right]=\mathrm{n}+1$.

$$
\text { (i) } \mathrm{i}\left[\mathrm{T}\left(\mathrm{H}_{\mathrm{n}}\right)\right]=2 \text { (ii) } \Delta\left[\mathrm{T}\left(\mathrm{H}_{\mathrm{n}}\right)\right]=2 \mathrm{n}
$$$$
\text { (iii) } \delta\left[\mathrm{T}\left(\mathrm{H}_{\mathrm{n}}\right)\right]=2
$$

\section{Observation 3.16}

(i)Every dominating set is a $(1,2)$-dominating set in $\mathrm{T}\left(\mathrm{H}_{\mathrm{n}}\right)$.

(ii)Every blast dominating set is a $(1,2)$-dominating set in $\mathrm{T}\left(\mathrm{H}_{\mathrm{n}}\right)$

\section{Theorem 3.17}

For any Fan graph $\mathrm{F}_{\mathrm{n}}(\mathrm{n} \geq 3), \gamma_{(1,2) c}^{t c}\left[T\left(F_{1, n}\right)\right]=\left\lceil\frac{n}{2}\right\rceil+1$

Proof

By the definition of total graph each edge $\mathrm{vu}_{\mathrm{i}}$ for $1 \leq \mathrm{i} \leq$ $\mathrm{n}$ of $F_{1, n}$ is subdivided by a new vertex $v_{i}$ and for $i=$ $1,2,3 \ldots . n-1, u_{i} u_{i+1}$ is subdivided by a vertex $w_{i}$.

Define $\quad V\left(T\left(F_{1, n}\right)\right)=\{v\} \cup\left\{v_{i}^{\prime}: 1 \leq i \leq n\right\} \cup$
$\left\{w_{i}: 1 \leq i \leq n-1\right\} \cup\left\{u_{i}: 1 \leq i \leq n\right\}$. Since $\quad v \cup\left\{v_{i}^{\prime}: 1 \leq\right.$ $i \leq n\}$ induces a clique of order $n+1$ in $T\left(F_{1, n}\right)$. The apex is adjacent to all the other vertices of $F_{1, n}$ and it dominates all the vertices of $T\left(F_{1, n}\right)$ except the vertex $w_{i}$. The induced 
graph $v_{i}^{\prime}$ is adjacent to all the vertices of $v_{i+1}^{\prime}$ also joining to $u_{i}$ and $w_{i}$ Let us consider $D=v \cup\left\{u_{2 i}\right\}$, whose induced subgraph $\langle D\rangle$ is connected dominating set and simultaneously, the induced subgraph of its complement $<V-D>$ is triple connected in $T\left(F_{1, n}\right)$. It has been proved that blast domination number exists for $T\left(F_{1, n}\right)$.

In $\mathrm{T}\left(F_{1, n}\right)$, fix as $v_{i}^{\prime}$ there exists a first vertex $v$ at distance 1 and second vertex $w_{i}$ or $u_{i+1}$ is at distance atmost 2 in $D$. So this will form a $(1,2)-$ blast dominating set. Now the presume result $\mathrm{D}=v \cup\left\{u_{2 i}\right\}$ holds. Therefore, the minimum (1,2)- Blast dominating set of the total graph of Fan graph is $\left\lceil\frac{n}{2}\right\rceil+1$

Result 3.18

For the total graph of Fan graph of order, $\mathrm{n} \geq 3$. Then (i) $\mathrm{i}\left[\mathrm{T}\left(\mathrm{F}_{1, \mathrm{n}}\right)\right]=\mathrm{n}-1$ (ii) $\Delta\left[\mathrm{T}\left(\mathrm{F}_{1, \mathrm{n}}\right)\right]=2 \mathrm{n}$

(iii) $\delta\left[\mathrm{T}\left(\mathrm{F}_{1 . \mathrm{n}}\right)\right]=4$

Theorem 3.19

Let $\mathrm{K}_{\mathrm{m}, \mathrm{n}}$ be a Complete Bipartite graph on $\mathrm{m}$ and $\mathrm{n}$ vertices respectively. Then $\gamma_{(1,2) \mathrm{c}}^{\mathrm{tc}}\left[\mathrm{T}\left(\mathrm{K}_{\mathrm{m}, \mathrm{n}}\right)\right]=3$

Proof

By the definition of total graph, let $\mathrm{v}_{\mathrm{ij}}$ be the newly in $\mathrm{T}\left(\mathrm{K}_{\mathrm{m}, \mathrm{n}}\right)$. Here $\mathrm{m}=2$. Let $\mathrm{V}\left[\mathrm{T}\left(\mathrm{K}_{\mathrm{m}, \mathrm{n}}\right)\right]=\left\{\mathrm{v}_{\mathrm{i}}: 1 \leq \mathrm{i} \leq\right.$ $m\} \cup\left\{u_{j}^{\prime}: 1 \leq \mathrm{j} \leq \mathrm{n}\right\} \cup\left\{\mathrm{v}_{\mathrm{ij}}: 1 \leq \mathrm{i} \leq \mathrm{m}, 1 \leq \mathrm{j} \leq \mathrm{n}\right\}$. Here the apex $v_{i}$ and $u_{j}$ are adjacent to each other. It incident with $\mathrm{m}$ and $\mathrm{n}$ edges.

Let us presume $\mathrm{D}=\left\{\mathrm{v}_{1}, \mathrm{u}_{1}, \mathrm{v}_{2}\right\}$ which is connected and the its complement is triple connected $\mathrm{T}\left(\mathrm{K}_{\mathrm{m}, \mathrm{n}}\right)$. Now, let us get the blast domination number of $\mathrm{T}\left(\mathrm{K}_{\mathrm{m}, \mathrm{n}}\right)=3$.

In $\mathrm{T}\left(\mathrm{K}_{\mathrm{m}, \mathrm{n}}\right)$ we see that the vertices in $\langle V-D\rangle$ there is a vertex which is of distance 1 and second vertex is of distance atmost 2.Thus it satisfies (1,2)-domination condition. Now the presume result holds for $\mathrm{T}\left(\mathrm{K}_{\mathrm{m}, \mathrm{n}}\right)$. Therefore, $\gamma_{(1,2) \mathrm{tc}}^{\mathrm{tc}}\left[\mathrm{T}\left(\mathrm{K}_{\mathrm{m}, \mathrm{n}}\right)\right]=3$.

Result 3.20

For any total graph of Complete Bipartite graph, here $(\mathrm{m}=2)$

$$
\text { (i) } \mathrm{i}\left[\mathrm{T}\left(\mathrm{K}_{\mathrm{m}, \mathrm{n}}\right)\right]=2 \text { (ii) } \Delta\left[\mathrm{T}\left(\mathrm{K}_{\mathrm{m}, \mathrm{n}}\right)\right]=2 \mathrm{n}
$$$$
\text { (iii) } \delta\left[\mathrm{T}\left(\mathrm{K}_{\mathrm{m}, \mathrm{n}}\right)\right]=\mathrm{m}+\mathrm{n}
$$

(iv) $\beta\left[\mathrm{T}\left(\mathrm{K}_{\mathrm{m}, \mathrm{n}}\right)\right]=3$.

Theorem 3.21

For any Comb graph $\mathrm{C}_{\mathrm{n}}, \gamma_{(1,2) \mathrm{c}}^{\mathrm{tc}}\left[\mathrm{T}\left(\mathrm{C}_{\mathrm{n}}\right)\right]=\mathrm{n}$

Proof

Let $\mathrm{G}$ be a graph with vertex set, $\mathrm{V}(\mathrm{G})=\mathrm{V}_{1} \cup \mathrm{V}_{2} \cup \mathrm{V}_{3}$, where $\mathrm{V}_{1}=\left\{\mathrm{v}_{\mathrm{i}}: 1 \leq \mathrm{i} \leq \mathrm{n}\right\}, \mathrm{V}_{2}=\left\{\mathrm{u}_{\mathrm{i}}: 1 \leq \mathrm{i} \leq \mathrm{n}\right\} \quad$ and $\mathrm{V}_{3}=\left\{\mathrm{v}_{\mathrm{i}}: 1 \leq \mathrm{i} \leq \mathrm{n}\right\} \cup\left\{\mathrm{u}_{\mathrm{i}}{ }^{\prime}: 1 \leq \mathrm{i} \leq \mathrm{n}-1\right\}$. In which the vertices $\mathrm{V}_{2}$ induces a path of order $\mathrm{n}$ and the vertex is adjacent to $\mathrm{V}_{2}$ and $\mathrm{V}_{3}$ of $\mathrm{T}\left(\mathrm{C}_{\mathrm{n}}\right)$ and it is also connected. Clearly $\mathrm{V}_{1}$ and $\mathrm{V}_{3}$ is triple connected. Thus we get $\mathrm{D}=\left\{\mathrm{V}_{2}\right\}$ forms a blast dominating set of $\mathrm{T}\left(\mathrm{C}_{\mathrm{n}}\right)$.

In $\mathrm{T}\left(\mathrm{C}_{\mathrm{n}}\right)$ we can note that the every vertex of $\mathrm{V}_{1}$ and $\mathrm{V}_{3}$ in $\mathrm{V}-\mathrm{D}$ there is atleast one vertex in $\mathrm{D}$ which is of at distance 1 and a second vertex which is of at distance introduced vertex in the edge connecting $\mathrm{v}_{\mathrm{i}}$ and $\mathrm{u}_{\mathrm{i}}$

atmost 2. It will forms a (1,2)- dominating set. Therefore, the minimum $(1,2)$ - Blast dominating set of total graph of Comb graph is $\mathrm{n}$.

Result 3.22

For any total graph of Comb graph, (here $\mathrm{m}=2)$

(i) $\mathrm{i}\left[\mathrm{T}\left(\mathrm{C}_{\mathrm{n}}\right)\right]=\mathrm{n}$

(ii) $\quad$ (ii) $\Delta\left[\mathrm{T}\left(\mathrm{C}_{\mathrm{n}}\right)\right]=5$

$$
\text { (iii) } \delta\left[\mathrm{T}\left(\mathrm{C}_{\mathrm{n}}\right)\right]=2
$$

\section{Observation 3.22}

Every $\gamma_{\mathrm{c}}^{\mathrm{tc}}$-set is a neighbourhood connected dominating set in the total graph of path, cycle, wheel, helm, fan, sunlet, complete bipartite and comb graphs.

Observation 3.23

Let $\mathrm{G}$ be a 3-regular graph on 8 vertices. Then $\gamma_{(1,2) c}^{t c}(G)=3$.

Exact values for some standard graphs

(i) For any Complete graph of order $n \geq 4$, we get $\gamma_{(1,2) c}^{t c}\left(K_{n}\right)=2$

(ii) For any wheel graph, $n \geq 3$ we have $\gamma_{(1,2) c}^{t c}\left(W_{n}\right)=2$

(iii) For any Fan Graph $n \geq 4$, we get $\gamma_{(1,2) c}^{t c}\left(F_{n}\right)=2$.

(iv) For any Complete Bipartite graph $n \geq 4$, we get $\gamma_{(1,2) c}^{t c}\left(K_{m, n}\right)=2$

\section{CONCLUSION}

In this paper, we computed the exact values of the $(1,2)$ blast domination number for some total graphs. In our further work, blast dominating set of Middle graphs of some more special classes of graphs are under investigation.

\section{REFERENCES}

1. Akbar, M.M., Panayappan, S. \& Vernold Vivin, J., Tulgeity of Line, Middle and Total graph of Wheel Families, International J.Math.Combin. Vol 3(2010), 98-107.

2. Chartrand, G \& Lesniak, L., Graphs and Digraphs, Chapman and Hall, CRC, 4th edition, 2005.

3. Haray, F., Graph Theory, Addison Wesley Reading Mass (1972).

4 Haynes,T.W., Hedetniemi, S.T. \& Slater, P.J., Fundamentals of domination in graphs, Marcel Dekker Inc. New York, U.S.A. (1998).

5 Mahadevan, G., Ahila, A., Selvam Avadayappan, Blast Domination Number for central and total graph of star, Global Journal of Pure and Applied Mathematics ,ISSN 0973-1768 Volume 13, No.2(2017).

6 Mahadevan, G., Ahila, A., Selvam Avadayappan, Blast Domination Number for $\vartheta$-Obrazom ,Int. Journal of Pure and Applied Mathematics ,Volume 118 No.4, 111-117(2018).

7 Murugesan, N and Deepa, S., Nair, (1,2)- Domination in Graphs, J.Math.Comput. Sci., Vol.2, 2012, No.4, 774783.

8 Paulraj Joseph , J., Angel Jebitha, M.K., Chithra Devi, P.\& Sudhana, G. Triple connected graphs, Indian Journal of Mathematics and Mathematical Sciences, Vol.8, No.1(2012),61-75. 
9 Sampathkumar, E. \& Walikar, H.B., The Connected Domination Number of a graph, J.Math.Phy.Sci.,13(6) (1979),607-613.

10 Steve Hedetniemi \& Sandee Hedetniemi, $(1,2)$ - Domination in Graphs.

11 Vernold Vivin, J., Harmonious Coloring of Total Graphs, nLeaf, Central graphs and Circumdetic Graphs, Ph.D Thesis,Bharathiyar University,(2007).

12 Vernold Vivin, J., Venkatachalam, M., On b-chromatic number of sunletgraph and wheel graph families, Journal of the Egyptian Mathematical Society (2015) 23, 215-218. 